\title{
Using Random Amplified Polymorphic DNA (RAPD) analysis to investigation of genetic diversity, and relationships among a set of clinical Aspergillus fumigatus isolates استخدام مؤشرات ال RAPD في تثخيص التغايرات الوراثية والعلاقة بين عزلات سريرية

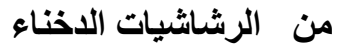

\section{Abstract}

This study is an attempt to determine the genetic diversity and relationships among fourteen local isolate isolated from patients with Aspergillosis (Aspergillus fumigatus) by using the Random Amplified Polymorphic DNA (RAPD) technique. Twelve universal primers used in this study produced 94 bands across fourteen isolates. Of these bands, 67 bands or $71.2 \%$ were polymorphic. The size of the amplified bands ranged between 100-2000 bp. The genetic polymorphism value of each primer was determined and ranged between 33-100\%. In terms of unique banding patterns, determine the finger print for six isolates the most characteristic banding pattern was for the (AFU1, AFU2, AFU3, AFU4, AFU8 and AFU14) with primer (OP F-16, OP I-06, OP F-16, OP X-01, OP X-01and OP A-06). Genetic distances ranged from 0.12419 to 0.64404 among $A$. fumigatus isolates. Cluster analyses were performed to construct a dendrogram among studied $A$. fumigatus isolates. The cluster analysis places most of the A.fumigatus isolates isolated from patient come from yhe same area into a close relation (subcluster) showing a high level of genetic relatedness and were distinct from isolates from another area (the other subcluster). Interestingly, a number of isolates originating from the same sources did form well defined groups, indicating association between the RAPD patterns and the geographic origin of the isolates. The information generated from this study can be used in the future for controlling of Aspergillosis programs.

A Keywords: DNA, RAPD, genetic distance, Aspergillus fumigatus.

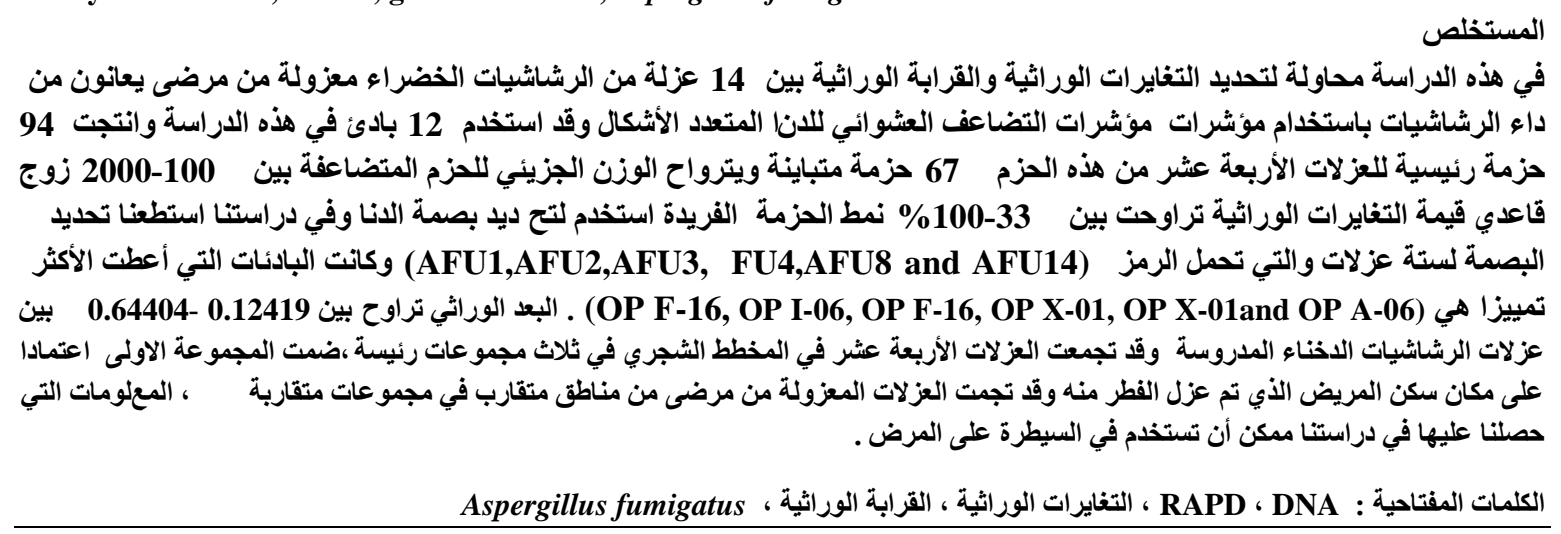
Introduction:

Aspergillus fumigatus is an opportunistic fungal pathogen responsible for most cases of aspergillosis, the most common systemic filamentous fungal infection worldwide [1]. The incidence of aspergillosis has increased during the last two decades [2] and saase [3,4]. 
Infection of A. fumigatus occurs by inhaling conidia which may colonize airways prior to invasion. In recent years, several molecular methods have been developed to explore the genetic diversity of A. fumigatus, including random amplified polymorphic DNA (RAPD), RAPD analysis can be performed quickly, a large number of isolates can be analyzed, and it can have a high discriminatory power. [5,6,7,8].

RAPD analysis assays DNA sequence variation in short regions using short primer and a low annealing temperature to generate several fragments in one amplification reaction[9]. Moreover, RAPD analysis is technically simple and often detects variation among isolates [10].

A better understanding of the role of environmental sources of A.fumigatus isolates causing infection would facilitate prevention strategies [11]. Clinical and environmental isolates could contribute to the solution of several relevant clinical problems such as the identification of the environmental source of A. fumigatus isolates causing aspergillosis, determining the existence of pathogenic isolates and the role of natural habitants, and also providing a guide for patient management [12].The aim of the present study investigation of Genetic Diversity, and Relationships among a Set of clinical A. fumigatus isolates .

\section{Materials and methods:}

\section{A. fumigatus isolates}

A total of 14 A. fumigatus isolates isolated from patients suffering from Aspergillosis, (as clinically identified by a physician), from Chest and respiratory diseases specialized center, Ministry of health Baghdad governorate (this specialized center generally accepted patients from different Iraqi governorate). all Isolates identifications were made on the basis of morphological and microscopical features, and were subcultured on Sabouraud Dextros Agar medium at $4 \mathrm{oC}$, for using it in DNA extraction A. fumigatus isolates isolated from patients comes from 1,2,3,4 (Baghdad) , 5 (Salahaldeen), 6 (Basrah) , 7,8 (Kerbala), 9,10 (Najaf), 11,12 (Dyala), 13(Wasit) , 14(Sulaimania) .

\section{Genomic DNA Isolation}

Total genomic DNA of all the studied isolates was extracted manually using CTAB method according to the method described by [13].to produce a rapid extraction and high quality extracted DNA. Purity and concentration of DNA was measured by spectrophotometer [14]. Genomic DNA integrity was detected by running on $1 \%$ agarose gel electrophoresis followed by staining with ethidium bromide and visualized under UV light [15]. DNA samples were diluted to a working concentration of $50 \mathrm{ng} / \mu \mathrm{l}$ in order to be use in the RAPD-PCR experiments.

\section{Primer selection and RAPD assay}

Twelve decamers of oligonucleotides primers random sequence were used (Operon Technologies) in a lyophilized form and were dissolved in sterile deionizer distilled water to give a final concentration of $(10 \mathrm{pmol} / \mu \mathrm{l})$ as recommended by provider the primers were tested in this study:(OP X-01, OP F-16, OP A-06, OP E-20, OP D-20, OP Y-03, OP I-06, OP E-16, OP Q-06, OP Q-01, OP A-10, OP N-07)

Amplification reactions were performed in a volume of $25 \mu l$ containing $5 \mu 1$ of PCR Master Mix (Bioneer), with concentration $(1 \mathrm{X})$ containing $(10 \mathrm{mM}$ Tris- $\mathrm{HCl}(\mathrm{pH} 8.3), 50 \mathrm{mM} \mathrm{KCl}, 1.5 \mathrm{mM} \mathrm{MgCl} 2,200 \mu \mathrm{M}$ each deoxynucleotide triphosphate (dNTP) and 1 unit DNA polymerase), 10pmol of the primer, and $50 \mathrm{ng}$ of template DNA. Amplification was carried out using a thermocycler (Eppendorf-Germany), using the following program:- 1 cycle of $5 \mathrm{~min}$ at $94^{\circ} \mathrm{C}$ for initial strand separation, followed by 45 cycles of $1 \mathrm{~min}$ at $94^{\circ} \mathrm{C}$ for $72^{\circ} \mathrm{C}$ for primer extension. Finally, 1 cycle of $10 \mathrm{~min}$ at $72^{\circ} \mathrm{C}$ was used for the final extension, followed by a hold at $4^{\circ} \mathrm{C}$ [16]. Each PCR amplification reaction was repeated twice to ensure reproducibility.

Twelve micro liter of PCR products were analyzed by electrophoresis in a $1.2 \%$ agarose gels at $5 \mathrm{Volt} / \mathrm{cm}$ for 2 hour in $0.5 \times$ TBE buffer, agarose gels were stained with ethidium bromide $0.5 \mu \mathrm{g} / \mathrm{ml}$ for $20-30$ minutes. The 100bp DNA ladder (100-2,000) bp (Bioneer) was used as a molecular size marker. After electrophoresis, images of gels were captured using Gel Documentation System (Consort - Belgium).

Data analysis

Molecular Weight Estimation 
Molecular weight was calculated by using the computer software M.W. detection program, Photo-Capture M.W. program from Consort, based on comparing the RAPD-PCR products with the known size of DNA fragments of a 100bp DNA ladder (which consist of 13 bands from 100 to 2,000 bp Bioneer).

\section{Estimation of Genetic Distances}

Data generated from the detection of polymorphic fragments were analyzed. The amplification profile of all the used isolates for any given primer were compared with each other, the presence of band scored as "1" and the absence of the same band of the same size in other isolate scored as "0".Only clear and reproducible amplified fragments were considered for genetic relationship analysis. Estimates of genetic distance (G.D) were calculated between all pairs of the varieties according to [17]. Based on following formula: G.D = 1$\{2 \mathrm{Nab} /(\mathrm{Na}+\mathrm{Nb})\}$

Where $\mathrm{Na}=$ the total number of fragments detected in individual ' $\mathrm{a}$ '; $\mathrm{Nb}=$ the total number of fragments shown by individual ' $b$ ' and $\mathrm{Nab}=$ the number of fragments shared by individuals 'a' and ' $b$ '. Cluster analysis was performed to construct genetic relationship tree diagrams among studied A.fumigatus isolates using an Unweighted Pair-Group Method with Arithmetic Average (UPGMA). All computations were carried out using the Numerical Taxonomy and Multivariate Analysis System (NTSYS-pc), Version 1.7 package [18]. The percentage of polymorphic bands was defined as ratio of the number of polymorphic bands amplified by a single primer to that of the total number of bands produced by the same primer.

\section{Results and discussion:}

The genetic diversity and the relationships among A.fumigatus isolates were evaluated using RAPD markers amplified from Twelve universal primers each of the primers varied greatly in their ability to resolve variability among isolates. Some primers generated several bands, while others generated only a few bands. Figure (1:A and B ).

A total of 94 useful bands were scored from the amplification products with the twelve random primers of DNA from 14 A.fumigatus isolates.

The number of amplification products generated by each primer varied from 6 (OP X-01) to 11 (OP Q-01) and ranged in size from $200 \mathrm{bp}$ (OP Y-03) to $2000 \mathrm{bp}$ (OP Q-01). In general, sufficient polymorphism existed to allow distinction between the isolates tested with, polymorphism ranged between (33-100\%), primer OP E-20, OP Q-01 and OP N-07produced the highest percent of polymorphism compared with primer OP X-01 Of these 94 PCR products generated, 29\% (27 bands) were monomorphic across all varieties, Many bands appeared in most of the varieties and were absent in only a few isolates. The remaining 67 bands $71 \%$ of the total products scored) were polymorphic among the studied isolates . A total of 67 (71.3\%) polymorphic bands were observed, ranging from 8 (OP I-06) to 58 (OP E-20) bands with an average of (5.5) polymorphic bands per primer across all the 14 A.fumigatus isolates.

This discrepancy may relate to the isolates and the selection of RAPD primers, The arbitrary primers (OP N07, OP Q-01, OP E-20) was the most useful for discriminating varieties of distinct characteristics. 


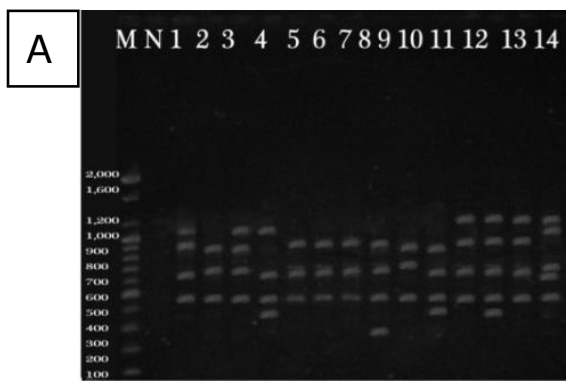

primer OP X-01

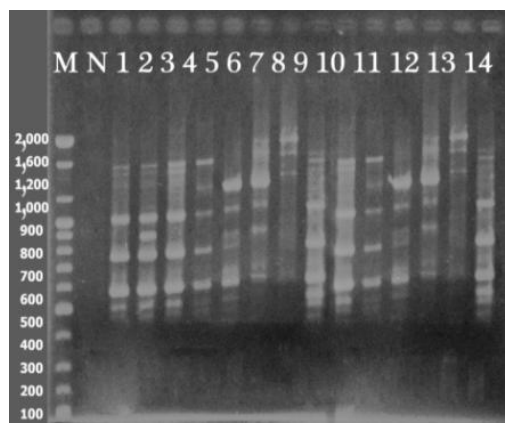

primer OP E-20.

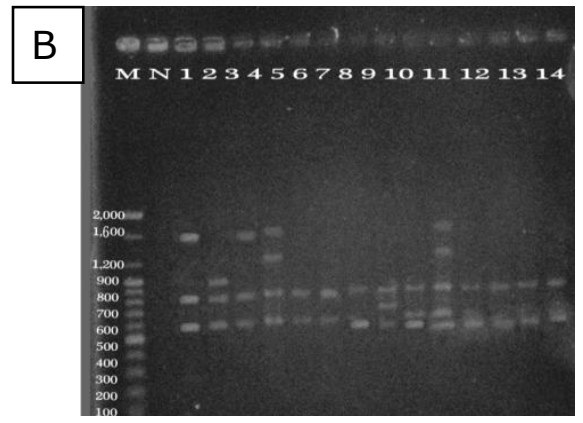

primer OP I-06

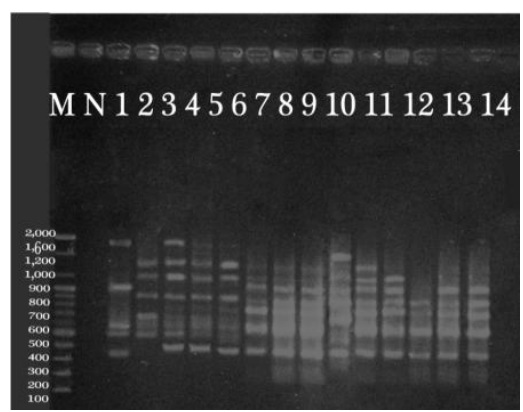

primer OP Q-01

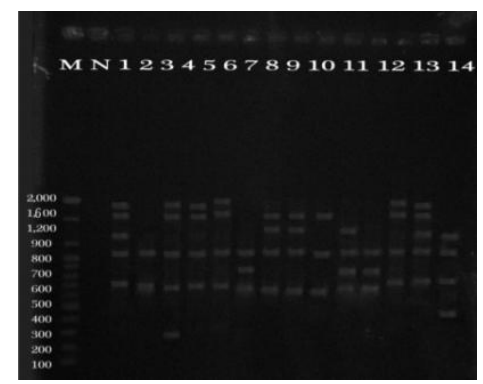

primer OP F-16

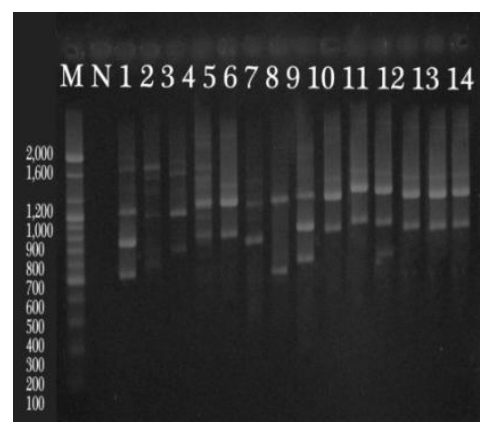

primer OP D-20

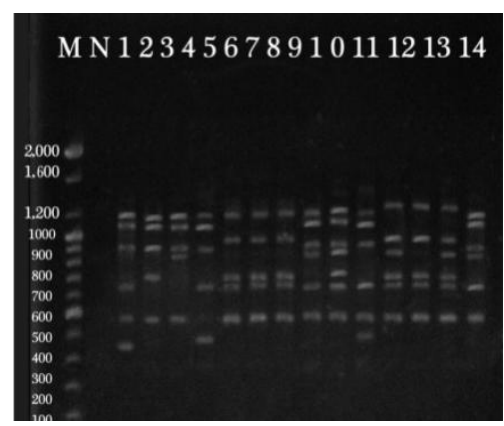

primer OP E-16

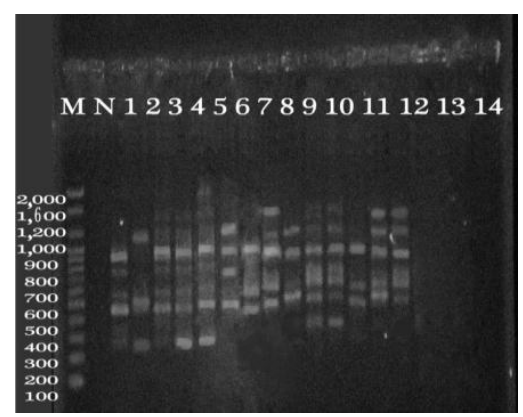

primer OP A-10

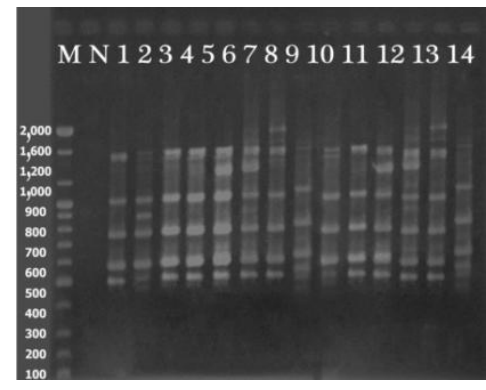

primer OP A-06

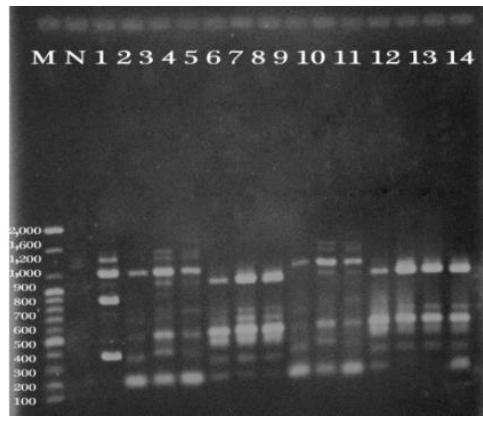

primer OP Y-03

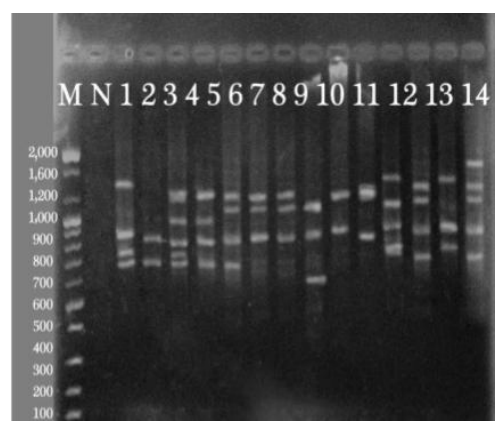

primer OP Q-06

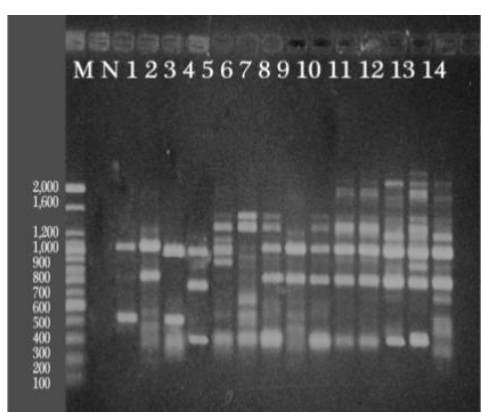

primer OP N-07

Figure (1:A and B ): Agarose gel electrophoresis of RAPD-PCR reaction All primers, DNA samples of A.fumigatus isolates (under optimal conditions). Bands were fractionated by electrophoresis on a 1.2\% agarose gel (2hr, $5 \mathrm{~V} / \mathrm{cm}, 1 \mathrm{XTris}-$ borate buffer) and visualized under U.V. light after staining with ethidium bromide. M: 100 bp DNA ladder. N: negative control. Lanes : A. fumigatus isolates from patients comes from : Lanes 1,2,3,4 (Baghdad) , Lanes:5(Salahaldeen) , Lanes:6 (Basrah) , Lanes: 7,8 (Kerbala) , Lanes:9,10 (Najaf), Lanes:11,12(Dyala) , Lanes:13(Wasit), Lanes:14(Sulaimania) .

Six of A.fumigatus isolates were distinguished by different primers in this study which produced the most important unique markers such as the isolate number ' 1 ' was distinguished by using primer (OP F-16 
$(1,200) b p$, OP D-20(2,000)bp, OP Y-03(2,000)bp, OP E-16 (400)bp, OP Q-06(1,600)bp), isolate number '2' was distinguished by using primer (OP I-06 $(1,000) \mathrm{bp}$ ), isolate number " 3 " by using primer (OP F-16 (300)bp), isolate nmber " 4 " was distinguished by using primer (OP X-01(400)bp) both of (OP X-01 (300)bp and OP Q-06 (600)bp) primers were distinguished the isolate number "8", while the isolates number "14" was distinguished by primer (OP A-06 (1,200bp) ) and (OP A-06(700bp) Thus, with selected primers sufficient polymorphism could be detected to allow identification of individual isolates, In most cases, the isolates with number " 3 and 4 " , "6 and 8", " 11 and 12", "9 and 10 " were genetically related, although clear differences between them could be seen.

\section{Genetic Distances}

The ratio of genetic similarity among the A.fumigatus isolates ranged from 0.35 to 0.87 table (1).The highest similarity (0.87) $87 \%$ was obtained between the isolate number '11' and '12'. This was followed by (0.79) $75 \%$ similarity between a pair of the isolates number ' 3 and 4 '. The lowest level of similarity $(0.36) 0 \%$ was obtained between the isolates number ' 2 ' and ' 11 '.

In this study, the value of genetic similarity is relatively agree when compared to the reports of other RAPD studies genetic similarity among clinical isolate of A.fumigatus in different area [19].

\section{Cluster analysis}

Dendrogram was constructed based on [17]. Genetic distance using UPGMA cluster analysis and depicted genetic relationships among 14 A. fumigatus isolates, showing four major clusters I, II ,III and IIII Figure (2). As expected all introduced isolates: the isiolate number " $1,3,4,2$ " were grouped into a cluster , including three subclusters, and other isolate with number" $5,11,12,13,6$ " were grouped into a cluster, including three subclusters, the third clusters the isolates " $7,8,9,10$ "were grouped into a cluster including two subclusters. The fourth major clusters including the isolate number " 14 " only .These fourth main group link together finally as shown in the figure (2).

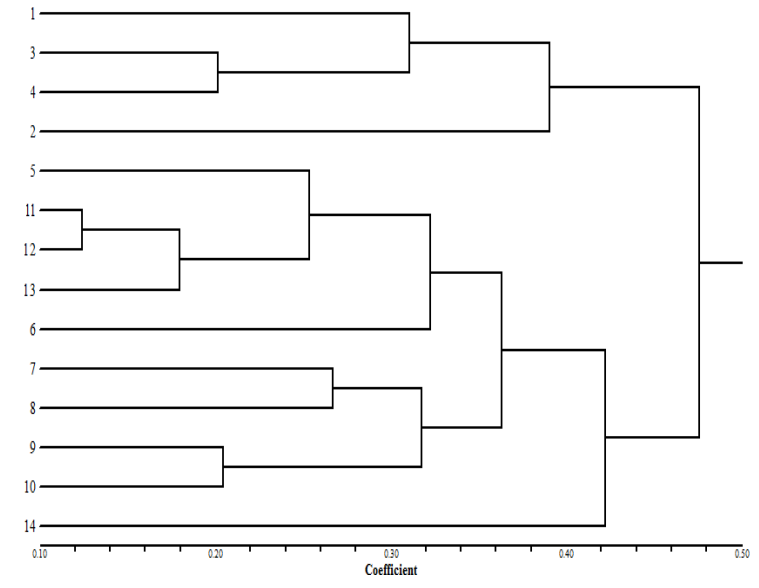

Fig (2): Dendrogram illustrated genetic fingerprint and relationships between A.fumigatus isolates developed from RAPD data .

A. fumigatus isolates from patients comes from : 1,2,3,4 (Baghdad) , 5(Salahaldeen) , 6 (Basrah) , 7,8 (Kerbala) , 9,10 (Najaf), 11,12(Dyala), 13(Wasit), 14(Sulaimania) . 
Table(1): Values of genetic distance between $A$. fumigates isolates calculated according to[17]

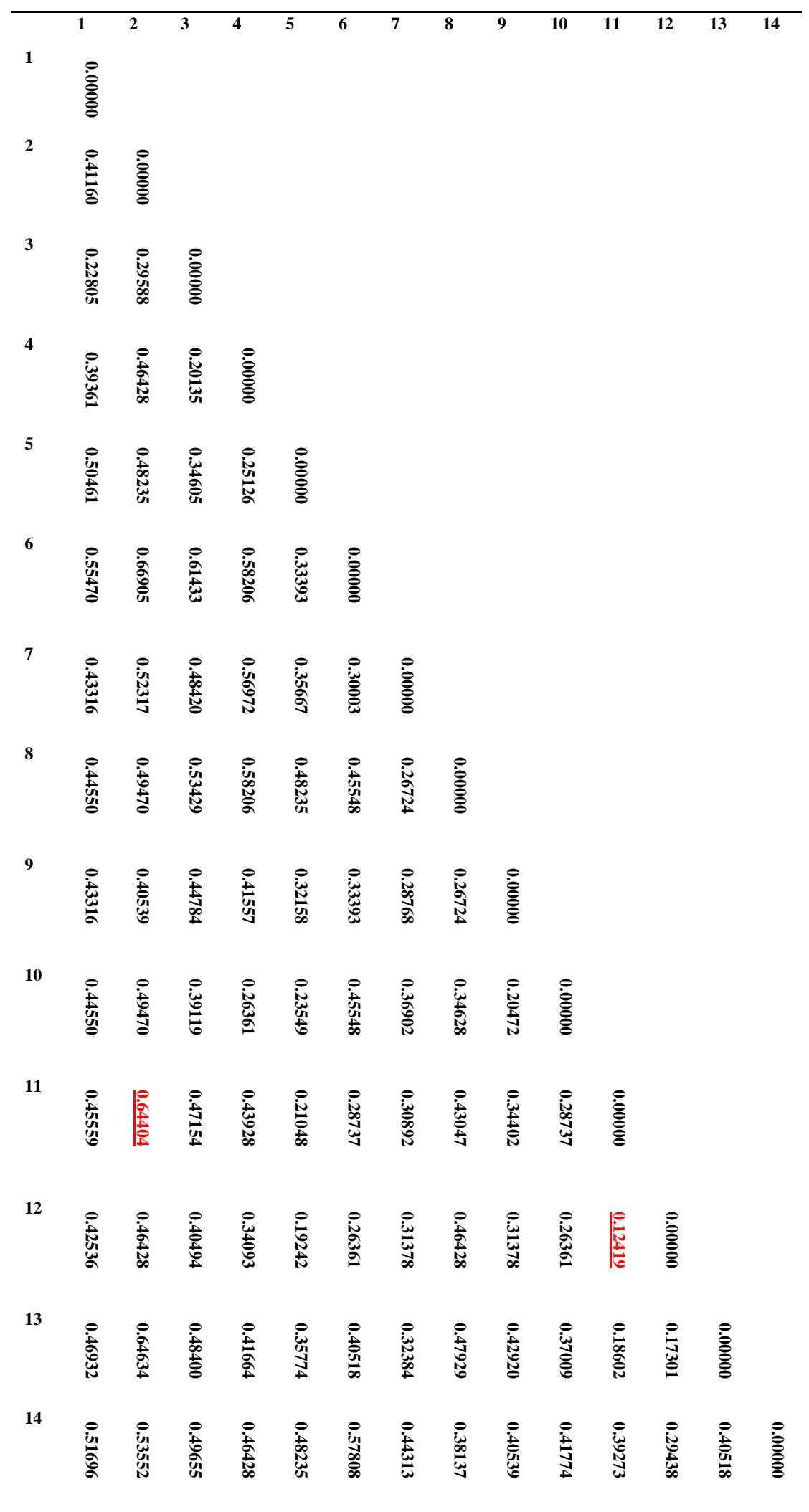


In this analysis, the isolate number "14" appeared to be genetically distinct due to this isolate isolated from patient comes from $\mathrm{Al}$ sulaimania the lower level of the similarity with all other isolate. Therefore, it formed a separate group (IV group).

Cluster analysis has placed most of the A. fumigatus isolates isolated from patients from the same goverment together, showing a high level of genetic relatedness and these were distinct from those isolates isolated from another government.

the dendrogram indicates a clear pattern of division among the A. fumigatus based on geographic origin of the isolates. Therefore, cluster analysis grouped the 14 isolates into four main clusters which correlated with their geographic origin

The analysis clearly distinguished among studied A. fumigatus. Such studies can be used to study genetic differences of isolates for their identification. Therefore, it might be predicted that RAPD may be effective in analyzing polymorphism at the A. fumigatus isolates, In the present study RAPD markers provided sufficient resolution to distinguish closely related isolates.

The information generated from this study gives a clearer picture of their genetic relationship and might possibly be developed into a standard classification procedure in the future and will be used in identifying efficient strategies for the controlling of aspergillosis caused by A. fumigatus in different governorates in Iraq.

The genetic distance between the isolates in our study may be due to that A. fumigatus is morphologically more variable $[20,21]$ than was originally described by [22].These variations have led to the description of several varieties of A. fumigatus, including acolumnaris, phialiseptus, ellipticus, and sclerotiorum, with the distinctions being based on onlyslight morphological differences. A. fumigatus, A. brevipes, A.duricaulis, A. unilateralis, A. viridinutans, together with anamorphs of species within the perfect genus Neosartorya, a genus in which morphologically related species have been grouped, are classified as Aspergillus sect. fumigati. The search for a sexual stage of A. fumigatus has been attempted among Neosartorya species, since it would allow classical genetics to be pursued in A. fumigatus. To date, no such stage has been discovered.

The benefits of using DNA-based typing techniques for the study of the genetic diversity of clinical isolates, revealing sources of infection, and for contributing to the solution of several relevant epidemiological problems of A. fumigatus linked isolates [23].An ideal technique should provide sufficient discrimination for the strains isolated from the epidemiological study of nosocomial outbreaks or from individual patients and their environment. However, only a limited number of the methods are useful for type A. fumigatus isolates. RAPD-PCR has been examined as a typing system for $A$. fumigatus typing due to both its usefulness and its relative technical simplicity and speed $[24,25,26,27]$.The major advantage of RAPD is that no previous sequence information is needed in contrast with other techniques like RFLP or microsatellite typing [23].

One of the applications of a typing method, such as RAPD, is to establish whether both clinical isolates collected from one distinct area are related. Nevertheless four identical electrophoretic banding patterns were observed with all clinical and environmental A. fumigatus isolates Therefore, it appears as if a close relationship exists between clinical and environmental isolates, suggesting that the environmental strains may responsible for causing invasive aspergillosis and that RAPD-typing can be a good approach for monitoring the source of infection. Although the extensive genetic diversity within $A$. fumigatus isolates makes it difficult to ensure the determination of area or hospitale or environment is the sources of infection, the isolation of the identical strains from a patient and from the hospital environment indicates that the infection was nosocomially acquired from the environment.this result agree with result of [28].

RAPD-PCR analysis can be applied as a simple, rapid, and useful method, and can also play a remarkable role in typing and differentiating A. fumigates isolates.

\section{Conclusion:}

In conclusion, RAPD analysis is a truly rapid and reliable tool in DNA fingerprinting. RAPD analysis is asensible and accurate method for epidemiologic studies of clinical outbreaks of A. fumigatus making use of the habitual techniques available in a current clinical microbiology laboratory. 


\section{Reference:}

1. Fridkin, S. A., and Jarvis, W.R. (1996). Epidemiology of fungal nosocomial infections. Clin. Microbiol. Rev. 9:499-511.

2. Rogers, T. R. (1995). Epidemiology and control of nosocomial fungal infections.Curr. Opin. Infect. Dis. 8:287-290.

3. Denning, D. W. (1998). Invasive aspergillosis. Clin. Infect. Dis. 26:781-803.

4. Wald, A., Leisenring, W., Van Burik, J. A. and Bowden, R. A. (1997). Epidemiology of Aspergillus infections in a large cohort of patients undergoing bone marrow transplantation. $\mathrm{J}$. Infect. Dis. 175:1459-1466.

5. Anderson, M.J., Gull, K. and Denning, D.W. (1996). Molecular typing by random amplification of polymorphic DNA and M13 Southern hybridization of related paired isolates of Aspergillus fumigatus. J. Clin. Microbiol. 34:87-93.

6. Lin, D., P. F. Lehmann, B. H. Hamory, A. A. Padhye, E. Durry, R. W. Pinner, and B. A. Lasker. (1995). Comparison of three typing methods for clinical and environmental isolates of Aspergillus fumigatus. J. Clin. Microbiol. 33:1596-1601.

7. Rodriguez, E., F. Symoens, P. Mondon, M. Mallie', M. A. Piens, B. Lebeau, A. M. Tortorano, F. Chaib, A. Carlotti, J. Villard, M. A. Viviani, F. Chapuis,N. Nolard, R. Grillot, and Bastide J. M. (1999). Combination of three typing methods for the molecular epidemiology of Aspergillus fumigatus infections.J. Med. Microbiol. 48:181-194.

8. Verweij, P. E., J. F. Meis, J. Sarfati, J. A. Hoogkamp-Korstanje, J. P. Latge',and W. J. Melchers. (1996). Genotypic characterization of sequential Aspergillus fumigatus isolates from patients with cystic fibrosis. J. Clin. Microbiol.34:2595-2597.

9. Wetton JH, Royston EC, Parkin DT, Walters D. (1987). Demographic study of a wild house sparrow population by DNA fingerprinting. Nature. 326:147-9.

10. Welsh J, McClelland M. (1990). Fingerprinting genomes using PCR with arbitrary primers. Nucleic Acid Research. 18:7213-8.

11. Lin D, Lehmann P, Fhamory, BH. (1995). Comparison of Three Typing Methods for Clinical and Environmental Isolates of Aspergillus fumigatus. J Clin Microbiol. 33 (6): 1596-1601.

12. Anderson MJ, Gull K, Denning DW. (1995). Molecular typing by Random Amplification of Polymorphic DNA and M13 Southern Hybridization of Related Paired Isolates of Aspergillus fumigatus. J.Clin.Microbiol 1995; 34 (1): 87-93.

13. Weigand, F., Baum, M. and Udupa, S. (1993). DNA molecular marker techniques, technical munual. No.20. international center for agricultural research in the dry area. alppo, Syria.

14. Sambrook, J., Friteg, E. F. and Maniatis, T. (1989). Molecular cloning : alaboratory . new york, USA.

15. Maniatis, T, Fritch, E.F. and Sambrook, J. (1982). Molecular cloning : Alboratory, New york.

16. Rabbani, MA, Pervaiz, ZH and Masood, MS. (2008). Genetic diversity analysis of traditional and improved cultivars of Pakistani rice (Oryza sativa L.) using RAPD markers. Molecular Biol and Genetics. 11(3)

17. Nei, M and Li, W. (1979). Mathematical model for studying genetic variation in terms of restriction endonucleases. Proc National Academy of Sci, (USA) 76(10): 5269-5273.

18. Rohlf, FJ. (1993). NTSYS-PC: Numerical Taxonomy and Multivariate Analysis System. Version 1.7. Applied Biostatistics Inc., Steauket, New York, USA.

19. Bertout, S., Renaud, F., Barton, R., Symens, F. Burnod, J., Piens, M., Lebeaus, M., Chapus, F., Bastide, M., Grilliot, R., and Lebeau, B. (2001). Genetic Polymorphism of Aspergillus fumigatus in Clinical Samples from Patients with Invasive Aspergillosis: Investigation Using Multiple Typing Methods. Clnical microbiology, p. 1731-1737 Vol. 39, No. 5.

20. Leslie, C. E., B. Flannigan, and L. J. R. Milne. (1988). Morphological studies on clinical isolates of Aspergillus fumigatus. J. Med. Vet. Mycol. 26:335-341.

21. Samson, R. A. (1994). Current systematics of the genus Aspergillus, p. 261-276. In K. A. Powell, A. Renwick, and J. F. Peberdy (ed.), The genus Aspergillus: from taxonomy and genetics to industrial application. Plenum Press, London, United Kingdom.

22. Puente, P., M. C. Ovejero, N. Fernandez, and F. Leal. (1991). Analysis of Aspergillus nidulans conidial antigens and their prevalence in other Aspergillus species. Infect. Immun. 59:4478-4485.

23. Varga, J. (2006). Molecular typing of aspergilli: Recent developments and outcomes. Medical Mycology.44 (S1): 149 - 161.

24. Anderson, MJ., Gull, K., Denning, DW. (1995). Molecular typing by Random Amplification of Polymorphic DNA and M13 Southern Hybridization of Related Paired Isolates of Aspergillus fumigatus. Clin.Microbiol. 34 (1): 87-93. 
25. Aufauvre-Brown, A., Cohen, J., Holden, DW. (1992). Use of Randomly Amplified Polymorphic DNA Markers to Distinguish Isolates of Aspergillus fumigatus. Clin Microbiol. 30 (11): 29912993.

26. Loudon, KW., Burnie, JP., Coke, AP., Matthews, RC. (1993). Application of polymerase chain reaction to fingerprinting Aspergillus fumigatus by random Amplification of polymorphic DNA. Clin Microbiol. 31: 1117-1121.

27. Treviño-Castellano, M., Rodríguez-Nóvoa, S., Llovo Taboada, J. (2003). Combined used of RAPD and touchdown PCR for epidemiological studies of Aspergillus fumigatus. Enferm Infecc Microbiol Clin. 21 (9): 472-6.

28. Varga, J. (2006). Molecular typing of aspergilli: Recent developments and outcomes. Medical Mycology. 44 (S1): 149 - 161. 